\title{
Discapacidad mental y ciudadanía activa: El desafío de una nueva legislación de salud mental para Chile ${ }^{1}$
}

\section{Mental disability and active citizenship: The challenge of a new legislation of mental health for Chile}

\author{
Ruben Nilo Pérez ${ }^{2}$ \\ Universidad de Santiago de Chile, Santiago, Chile
}

(Rec: octubre 2014 - Acept: junio 2015)

\begin{abstract}
Resumen
El enfoque de la discapacidad constituye actualmente una perspectiva que pretende hacerse cargo de la incorporación de la dimensión social en la comprensión de la salud mental, y su hegemonía en Chile es visible en el diseño de las legislaciones y políticas públicas sobre salud mental durante los últimos 20 años. Sin embargo, tanto este enfoque de la salud mental como las respectivas legislaciones y políticas desarrolladas, presentan importantes dificultades para hacerse cargo de la situación de justicia y ciudadanía de este grupo de personas. Estas dificultades del enfoque se hacen particularmente evidentes cuando se requiere garantizar el ejercicio de una ciudadanía activa, ya que los aspectos relativos a la ciudadanía han demostrado tener un efecto terapéutico sobre las deficiencias o trastornos mentales. La urgente necesidad de una ley de salud mental para Chile demanda el desafío de diseñar una legislación que se haga cargo de las particularidades de la discapacidad mental, pero además la posibilidad histórica de incluir efectivamente la perspectiva de las personas con discapacidad mental y sus familias en la discusión de una nueva ley.
\end{abstract}

Palabras clave: discapacidad mental, ciudadanía activa, legislación en salud mental.

\begin{abstract}
Nowadays, the disability approach is a perspective that proposes a way to deal with the incorporation of social dimension in mental health understanding. Its hegemony in Chile turns visible considering the design of mental health laws and public policies over the past 20 years. However, this approach to mental health as well as the legislations and policies developed and related to it, hardly take over the situation of justice and citizenship of this group of people. These difficulties are particularly relevant when the exercise of active citizenship is required, since citizenship has been proved to show a therapeutic effect on the deficiencies or mental disorders. The urgent need of a mental health law for Chile demands the challenge of designing a law which recognizes the particularities of mental disability, but it also offers the historical possibility to effectively include the perspective of people with mental disabilities and their families in the discussion for a new law.
\end{abstract}

Keywords: mental disability, active citizenship, mental health legislation.

Este artículo fue desarrollado en el marco del proyecto PAI No 781403013, financiado por CONICYT

2 Correspondencia a: Ruben Nilo Pérez. Universidad de Santiago de Chile, Departamento de Historia, Av. Ecuador \#3363, Estación Central, Región Metropolitana de Santiago, Chile. E-mail: rubennilo@gmail.com. 


\section{Introducción}

En las últimas tres décadas hemos presenciado, desde el ámbito de la salud mental, la progresiva instalación de un enfoque o acercamiento a su objeto de estudio que pareciera haber alcanzado un estatus de paradigma, si consideramos su carácter hegemónico y su amplio efecto, visible en las políticas públicas y legislaciones relativas a la salud mental desarrolladas en Chile y otros países al alero de las orientaciones emanadas desde Naciones Unidas. Se trata del enfoque de la discapacidad, una nueva formulación del antiguo problema de la salud mental que pretende hacerse cargo de la incorporación de los aspectos sociales involucrados en la misma, de acuerdo a una pretendida superación del paradigma biomédico, que ha estado en ejercicio desde el "gran encierro" del siglo XVII (Foucault, 1967). Sin embargo, el enfoque de la discapacidad conceptualiza la discapacidad mental más bien como una consecuencia de la enfermedad o deficiencia mental para la vida de las personas, obviando el importante papel de los aspectos sociales en la etiología de las enfermedades mentales, pero permitiendo en cambio estimar linealmente el "grado" de esta discapacidad. La categorización y medición de la discapacidad es, en la actualidad, la base de las políticas públicas desarrolladas en Chile en el área de los trastornos mentales, así como de los avances legislativos desarrollados en la materia.

A casi treinta años de instalación del enfoque de la discapacidad, resulta necesario evaluar de qué manera dicho modelo ha facilitado cambios y avances efectivos en la vida de quienes padecen este tipo de discapacidad, así como de quienes trabajan y viven de manera cercana con esta realidad. Esta evaluación puede realizarse desde diversas aproximaciones considerando, por ejemplo, la cobertura y calidad de la atención de salud mental ofrecida por el Estado (la asistencia), o la situación de este grupo de personas en términos de sus derechos humanos y, particularmente, en términos de su participación activa en la vida social (la ciudadanía activa). Evaluar la pertinencia y eficacia de este enfoque para hacerse cargo del problema de la discapacidad mental es una tarea necesaria, pero que excede las posibilidades de este trabajo. Mi pretensión es más bien clarificar la situación de las personas con trastornos mentales al interior de este enfoque para argumentar que en lo referente a la situación de justicia de estas personas, el ejercicio efectivo de una ciudadanía activa no parece estar garantizado a nivel legislativo ni de políticas públicas, ni tampoco desde un enfoque conceptual que no se hace cargo del potencial terapéutico de las variables de tipo social en la etiología y tratamiento de las deficiencias o trastornos mentales.

Valga recordar que aquí se trata de un tipo de discapacidad particularmente sensible a los problemas de la justicia y la ciudadanía democrática, ya que estas consideraciones han probado sobradamente tener un efecto positivo en la evolución no sólo de la discapacidad que puede presentar una persona, sino también en la deficiencia psíquica que ha dado origen a su condición de discapacidad y minusvalía (Sarraceno, 2003). El importante peso que han mostrado tener las variables contextuales y el funcionamiento social de los individuos en la cura y/o rehabilitación de las enfermedades mentales nos permite afirmar que el ejercicio activo de su ciudadanía es, en su caso particular, algo no sólo justo sino también bueno, dado su carácter terapéutico.

La necesidad de crear una legislación de salud mental, para el caso de Chile, hace aún más necesario revisar no sólo los conceptos, sino la forma en que nuestra sociedad pretende hacerse cargo de un problema tan antiguo como vigente: las precarias condiciones de justicia que históricamente han debido enfrentar estas personas. En este sentido, las consideraciones de justicia respecto a las personas con discapacidad mental deben implicar una revisión de la noción de ciudadanía a la base de nuestras políticas y legislaciones específicas, ya que éstas parecieran excluir de dicha categoría, tanto en lo teórico como en lo práctico, a quienes padecen este tipo de discapacidades. Por lo demás, la exclusión de quienes padecen trastornos mentales de la condición de ciudadanía ha caracterizado históricamente la forma en que nuestras sociedades se han hecho cargo de esta problemática.

\section{Sobre la noción de "discapacidad mental"}

En términos generales, el enfoque de la discapacidad entiende actualmente la "deficiencia" como la pérdida de una función corporal normal, la "discapacidad" como lo que no podemos hacer en nuestro entorno por causa de la deficiencia y la "minusvalía" como la desventaja competitiva resultante a causa de la discapacidad (Nusbbaum, 2006; Sarraceno, 2003). Las discapacidades, por tanto, pueden ser originadas por deficiencias físicas, mentales, intelectuales o sensoriales, tal como lo establece la Convención sobre los Derechos de las Personas con Discapacidad, de carácter vinculante para los estados miembros (Organización de las Naciones Unidas, 2006), y como fue establecido el año 1993 por Naciones Unidas, al terminar el 
decenio. Resulta importante constatar, en este sentido, que la terminología de este enfoque ha sido utilizada en nuestro país de manera poco certera en el área de la salud mental, tanto en el diseño de políticas públicas como de legislaciones, confundiendo la terminología en uso para diferenciar la discapacidad mental (provocada por trastornos mentales) de la discapacidad cognitiva (originada por un déficit de tipo intelectual), aunque como bien sabemos ambas pueden presentarse juntas en la vida de una persona.

Al revisar las políticas públicas y la legislación actual sobre discapacidad vigente en nuestro país (Ley 20.422, 2015) vemos que se utiliza el concepto de "discapacidad mental" para hacer referencia tanto a las deficiencias psíquicas como a las intelectuales, homologando en la práctica estos tres conceptos y complicando enormemente la clarificación conceptual. Por su parte, el reglamento específico creado por el Estado para la calificación de la discapacidad (Ley 19.284, Decreto 2505, Aprueba reglamento para la Evaluación y Calificación de la discapacidad, 2004) propone la categoría unificada de "deficiencia psíquica o mental" para hacer referencia a quienes presentan deficiencias de tipo mental y/o intelectual, respectivamente, complicando aún más la tarea de definir con claridad los conceptos básicos. Este confuso panorama conceptual se torna francamente inverosímil cuando consideramos además lo establecido por la Ley 18.600 (Establece normas sobre deficientes mentales, 2008), único cuerpo legal específico sobre salud mental vigente en Chile desde 1987, y que establece que la persona con "discapacidad mental" es aquélla que presenta limitaciones o deficiencias de tipo "psíquico". En este contexto, es posible distinguir al menos que lo "psíquico" aparece en nuestra legislación actual en lugar de lo que los estándares internacionales entienden como "mental", concepto que en nuestra legislación refiere también a lo que estos estándares entienden como "cognitivo" o "intelectual".

Así las cosas, en el marco del presente trabajo he optado por utilizar el concepto de discapacidad mental para hacer referencia a lo que la Organización Mundial de la Salud y diversos organismos internacionales efectivamente entienden por "discapacidad mental", incluyendo aquéllos trastornos mentales o psiquiátricos severos que no implican, necesariamente, limitaciones de tipo cognitivo o intelectual (vale decir, un coeficiente intelectual inferior a 70 acreditado mediante un instrumento aprobado por la OMS), pero que tal como lo señala nuestra legislación implican "trastornos en el comportamiento adaptativo, previsiblemente permanentes" (Aprueba reglamento para la evaluación y calificación de la discapacidad, 2014). Para efectos comprensivos, valga señalar que el Servicio Nacional de la Discapacidad incluye en esta categoría patologías como el autismo, las psicosis de causa orgánica, la paranoia, la depresión bipolar y la esquizofrenia, aunque bien sabemos que estas categorizaciones son arbitrarias en ocasiones. También conceptualizadas como patologías psiquiátricas, se trata de trastornos que han sido caracterizados como severos por sus efectos discapacitantes, siendo la esquizofrenia un caso paradigmático en este sentido tal como lo establece nuestro Plan Nacional de Salud Mental y Psiquiatría (Ministerio de Salud de Chile, 2000).

La esquizofrenia tiene en Chile una prevalencia de 0,5\% en la población mayor de 15 años (Loubat, Gárate \& Cuturrufo, 2014). y se caracteriza por "distorsiones fundamentales y típicas de la percepción, del pensamiento y de las emociones, estas últimas en forma de embotamiento o falta de adecuación de las mismas" (MINSAL, 2000, p. 97), las que sin embargo no siempre están presentes en la totalidad de la vida de estas personas sino que pueden constituir una situación de tipo transitorio, no necesariamente recurrente. Siendo una de las siete prioridades programáticas establecidas por el Plan Nacional de Salud Mental y Psiquiatría, la esquizofrenia es entendida como un trastorno particularmente discapacitante ya que "compromete las funciones esenciales que dan a la persona normal la vivencia de su individualidad, singularidad y dominio de sí mismo" (MINSAL, 2000). Sin embargo, es necesario considerar que trastornos como la depresión pueden cumplir igualmente con la mayoría de estos criterios, siendo ésta el problema de salud mental que genera mayor discapacidad a las mujeres en nuestra país y ocupa el segundo lugar entre las causas de años de vida saludable perdidos por discapacidad o muerte prematura (AVISA) en ellas (MINSAL, 2000).

Más allá de estas distinciones, podemos convenir a estas alturas que el concepto de discapacidad mental sea utilizado en este trabajo para señalar a aquellos que históricamente han sido calificados desde el sentido común como "locos", "enfermos mentales" o "pacientes psiquiátricos". La distinción que deseo establecer es que, a pesar de los efectos negativos que este tipo de trastornos pueden tener en el funcionamiento cognitivo de quienes los presentan, las limitaciones intelectuales no constituyen una condición necesaria de la discapacidad mental y, por otra parte, la sintomatología presente en estas deficiencias puede desaparecer en algunos casos, ya sea de manera transitoria o permanente (lo cual 
no ocurre en el caso de la discapacidad intelectual). En este sentido, como ya se ha señalado la discapacidad mental presenta otro elemento que la distingue de las discapacidades de tipo físico y cognitivo: los desarrollos más recientes coinciden en que la intervención eficaz en la discapacidad y minusvalía mental tiene un efecto directo en la disminución de la deficiencia, reduciendo el daño que ha dado origen a la condición de discapacidad. Tal como lo ha señalado Sarraceno “...los síntomas (daños) del esquizofrénico pueden cambiar gracias a la intervención sobre su discapacidad y minusvalía" (Sarraceno, 2003, p. 15), por lo que en el caso de la discapacidad mental los aspectos referidos a la justicia social y la ciudadanía activa tienen no sólo una finalidad ética, sino terapéutica.

La clarificación conceptual resulta necesaria si queremos mirar críticamente la manera en que el enfoque de la discapacidad se hace cargo de los derechos de ciudadanía de este grupo de personas, y de su inclusión en la sociedad. Quienes han sido entonces incluidos en la categoría de discapacitado mental, son personas que presentan necesidades y recursos de muy distinto tipo, tanto entre ellos mismos como respecto a quienes padecen otros tipos de discapacidades. Definir con claridad esta categoría conceptual es una condición necesaria para cualquier discusión ya que implica asumir, al menos, 1) que las personas con discapacidad mental no presentan necesariamente deficiencias de tipo intelectual (cognitivo); 2) que la deficiencia (sintomatología) mental puede desaparecer de manera transitoria o permanente; y 3) que a diferencia de los otros tipos de discapacidad descritos, la intervención sobre los aspectos sociales de la discapacidad (inclusión y participación social) ha demostrado tener un efecto terapéutico (curativo) sobre la deficiencia o enfermedad mental que la ha originado.

\section{Discapacidad y enfoque de las capacidades}

Quienes presentan discapacidades mentales constituyen un grupo de personas que ha permanecido históricamente excluido del sistema social y de la participación activa en la comunidad, por causa de sus diferencias. Éstas han sido conceptualizadas como desviación, locura, enfermedad o discapacidad, de acuerdo al paradigma en uso sobre la salud mental. Durante el siglo XX se registró una evolución o tránsito desde un paradigma biologicista (centrado en la etiología y manifestaciones de la enfermedad), hacia un paradigma que actualmente entiende la salud como el ejercicio activo de determinadas capacidades humanas básicas, y que pone de relieve el papel de los aspectos sociales en la producción de la salud y la enfermedad. La incorporación de "lo social" y de las conceptualizaciones "positivas" sobre la salud ha ganado fuerza de manera sostenida en el desarrollo de las políticas públicas sobre la salud mental, siendo actualmente el paradigma "oficial" que organismos internacionales como la OMS y OPS utilizan para diseñar sus legislaciones y políticas públicas relativas a la salud mental (OMS, 2001; 2006; 2013).

Particular fuerza ha cobrado, en el contexto de este paradigma social de la salud, el enfoque de la "discapacidad", noción utilizada para referir a las consecuencias o impacto que una enfermedad o deficiencia provoca en la vida de una persona (Nussbaum, 2007; OMS, 2006). Se trata de un enfoque que persigue la superación del modelo biologicista de la salud, ya que separa aguas entre lo que correspondería al tratamiento médico de la enfermedad (deficiencia), y lo que correspondería a los aspectos sociales involucrados en el proceso de salud-enfermedad, particularmente en términos del impacto negativo de la deficiencia en la vida de las personas (discapacidad) (Toboso \& Arnau, 2008). Cabe puntualizar que las aproximaciones del enfoque de la discapacidad han generado adeptos y detractores, y las críticas han apuntado fundamentalmente a la persistencia de una conceptualización negativa a la base de la noción de discapacidad, y a la comprensión de los aspectos sociales como consecuencias y no como causas de los problemas de salud. Estas y otras objeciones fueron recogidas por la OMS en su Clasificación Internacional del Funcionamiento, de la Discapacidad y de la Salud (OMS, 2001), evidenciando un avance hacia una conceptualización positiva de la salud y a la inclusión de los aspectos sociales en todo el proceso o "estado de salud" de las personas. Sin embargo, estos desarrollos más bien técnicos no han tenido mayor efecto en las legislaciones y políticas públicas desarrolladas en Chile, las que han continuado nutriéndose de la conceptualización más clásica de este enfoque, que entiende la salud mental a partir de la nociones de deficiencia, discapacidad y minusvalía, y se ocupa preferentemente de las consecuencias de las enfermedades mentales para la vida de las personas.

\section{El Enfoque Capacidad de Amartya Sen}

Un análisis en profundidad del enfoque de la discapacidad (a la base de nuestros últimos avances 
legislativos), evidencia sus raíces más profundas en los planteamientos económicos de Amartya Sen (1992), y en sus desarrollos posteriores en el campo de la filosofía política y el derecho comparado, como es el caso de Martha Nussbaum (2007). La reflexión de Sen sobre los problemas de la desigualdad y el bienestar se articula en torno a la pregunta: ¿igualdad de qué?, originada en su constatación de que todas las teorías éticas actuales son "igualitaristas" en un sentido fundamental y que realmente sólo se distinguen entre sí por el acento que ponen en determinadas variables centrales que definen la igualdad, como por ejemplo las libertades individuales, los recursos, el ingreso o, en el caso de su propuesta alternativa, las capacidades de funcionamiento, vale decir, las competencias de los individuos para "realizar el tipo de vida que tienen razones para juzgar valioso" (Ovejero, Martí \& Gargarella, 2004, p. 55). De acuerdo a Sen, la limitación de los enfoques existentes sobre la igualdad surge cuando consideramos la diversidad intrínseca de lo humano, y el hecho que la satisfacción de ciertas igualdades (por ejemplo, de oportunidades) puede ser incompatible con la satisfacción de otras formas posibles de igualdad (por ejemplo, de ingresos). Se trata de un énfasis fundamental de su enfoque, ya que "la diversidad humana no es una complicación secundaria, que se pueda pasar por alto, o que hay que introducir "más tarde", sino un aspecto fundamental de nuestro estudio de la igualdad" (Sen, 1992, p. 9).

Para responder a la pregunta ¿igualdad de qué? Sen propone atender a la "libertad efectiva" que tienen las personas para realizarse, más que al abanico de libertades que se den por aseguradas para los ciudadanos, como es el caso de los bienes primarios en la teoría rawlsiana (Rawls, 1971; 2001). Dicha libertad efectiva es conceptualizada en su enfoque como una capacidad para la realización, mientras que la noción de funcionamiento señala la realización efectiva de nuestras ideas de lo bueno y nuestros proyectos de vida a través del uso de nuestras capacidades. La reflexión de Sen constituye una crítica explícita a la teoría de Rawls (si bien su análisis es también subsidiario de la misma, como el propio Sen lo reconoce), a la que acusa de centrar su atención en los medios (los bienes primarios de Rawls) para conseguir la igualdad deseada, más que en las capacidades reales que tienen las personas para realizar funcionamientos valiosos para su propio bienestar y calidad de vida (Sen, 1995). Su enfoque de la "capacidad" se orienta a la libertad misma y no a los medios para alcanzarla, en sus propias palabras "En la medida en que los funcionamientos son constitutivos del bien-estar, la capacidad representa la libertad de una persona para alcanzar el bienestar" (Sen, 1995, p. 63).

Sen subraya el problema de que los bienes primarios como el ingreso son sólo medios para alcanzar la libertad, y por tanto requieren ser "transformados" por las personas en libertad efectiva para la realización de diversos funcionamientos valiosos. Por tanto, una estimación lineal de la calidad de vida a partir de los ingresos como la propuesta por Rawls sería incompatible con la variabilidad de recursos y necesidades que tienen las personas, situación que resulta especialmente visible en el caso de las personas con algún tipo de discapacidad física o mental, quienes pueden poseer más bienes primarios que otras personas, pero al mismo tiempo menos capacidad debido a su minusvalía (Sen, 1995). Si bien Sen sugiere la posibilidad de que los ingresos pueden ser una medida adecuada de estimación de la desigualdad social (Nussbaum, 2007, exige como requisito previo considerar la diversidad de necesidades y recursos que presentan los sujetos para la realización de sus capacidades personales. No hacerlo implica ignorar desigualdades fundamentales que permanecerán, a pesar de que exista una distribución igual de los ingresos, bajo la forma de la discapacidad y la minusvalía.

La estimación de la desigualdad depende finalmente de la consideración de la diversidad de los seres humanos y de los distintos ámbitos en que se puede considerar la desigualdad, vale decir, de los bienes primarios que se utilicen como variables focales para la evaluación de la desigualdad. De esta manera, la pregunta por el sentido fundamental de la igualdad parece ser un falso problema en el enfoque de Sen, ya que en él "la igualdad se expresa de muchas maneras y cada sentido es pertinente según el contexto de evaluación" (Urquijo, 2007, p. 41). Lo relevante en cada caso es que el foco no está puesto en la distribución de los bienes primarios, sino en la capacidad o libertad efectiva de las personas para disfrutar de ellos.

\section{El enfoque de las capacidades de Martha Nussbaum}

Por su parte Martha Nusbbaum, filósofa política de orientación liberal y continuadora de la tradición de John Rawls desarrolla, a partir de los planteamientos de Amartya Sen en el campo de la economía, una teoría sobre la justicia que pretende explícitamente hacerse cargo del fenómeno de la discapacidad, entre otros problemas sociales que no han sido considerados por 
las teorías clásicas sobre la justicia, como la justicia internacional y los derechos de los animales no humanos (Nussbaum, 2007). A la manera de una ética de mínimos de justicia (Cortina, 1986) y en estrecha cercanía con el enfoque de derechos humanos, la asistencia y ciudadanía de quienes padecen estas discapacidades aparece garantizada en este modelo por una lista de capacidades humanas básicas que persigue garantizar un umbral mínimo para que la vida humana pueda ser vivida como tal. Valga señalar que esta lista es definida por la autora como "intuitiva" y "provisional", sujeta a correcciones y modificaciones pero necesaria para una teoría mínima de la justicia social. La lista actualmente formulada por Nussbaum (2007), comprende las 10 capacidades humanas básicas de: 1) Vida, 2) Salud Física, 3) Integridad Física, 4) Sentidos, Imaginación y Pensamiento, 5) Emociones, 6) Razón Práctica, 7) Afiliación, 8) Relación con Otras Especies, 9) Juego y 10) Control sobre el propio entorno.

Respecto a la asistencia de la discapacidad, Nussbaum señala que no es posible conceptualizarla como un bien primario más ni tampoco como una capacidad más en su lista, ya que la asistencia no constituye un hecho unitario. Retomando las ideas de Amartya Sen, defiende la idea de que a la variabilidad de los recursos y necesidades de los ciudadanos corresponde un enfoque individualizado de la asistencia que se ajuste a cada persona. Entendida de esta manera, la asistencia es concebida como un fenómeno transversal a todas las capacidades humanas de su lista, y por tanto como una necesidad humana básica cuya satisfacción es tarea prioritaria de toda la sociedad.

A pesar de la enorme contribución de las reflexiones de Nussbaum para la situación de justicia de quienes padecen discapacidades de tipo psíquico, considero que su enfoque de las apacidades continúa siendo insuficiente para la tarea de pensar una forma de la ciudadanía que garantice la inclusión de este grupo de personas en la vida social. Como he anticipado, este problema nos obliga a pensar una forma de ciudadanía que incluya efectivamente a estas personas, y en este sentido la lista de capacidades de Nussbaum, si bien propone un umbral mínimo de capacidades que operan también como criterios para la asistencia de la discapacidad mental (conceptualizada como un fenómeno transversal a éstas), no parece ofrecer una guía ni garantía clara para la deuda histórica de nuestras sociedades respecto a la participación activa de estas personas en la sociedad.

Si bien es posible distinguir un acercamiento a través de lo que ella denomina la capacidad de "control sobre el propio entorno", su descripción de esta capacidad parece poner el acento más bien en el ejercicio de derechos y libertades políticas (como el derecho a sufragio y las libertades de expresión y asociación) que en los aspectos relativos a la ciudadanía activa, vale decir, al ejercicio efectivo de sus capacidades de participación social y de influir sobre su entorno. Cabe recordar, que en los planteamientos de Amartya Sen estas preocupaciones ya aparecían esbozadas en su distinción conceptual entre capacidades y funcionamientos. En Nusbbaum, esta omisión aparece contradictoria con sus propias reflexiones, ya que resulta clara su preocupación porque la discapacidad sea vista no sólo como un problema de asistencia de salud, sino como un problema social, ya que finalmente es responsabilidad de la sociedad completa generar las adecuaciones y transformaciones necesarias para que este grupo de personas pueda llevar una vida efectivamente digna.

En este punto, considero posible sostener la hipótesis de que la insuficiencia del enfoque de las capacidades aparece determinada por su énfasis en las consecuencias o impacto de la discapacidad mental en quienes la padecen, más que en la consideración de los aspectos sociales de la discapacidad mental en la génesis y desarrollo de este tipo de trastornos o deficiencias. Tal como hemos señalado anteriormente, para el caso de las personas incluidas en esta categoría el foco debiese estar puesto precisamente en las capacidades relativas a la participación social y de realización efectiva de sus proyectos vitales, las que he incorporado bajo la noción de ciudadanía activa, con la sola pretensión de distinguirla de las nociones de ciudadanía de tipo declarativo o pasivas, que ponen el acento en el aseguramiento de derechos y libertades, pero no necesariamente en el uso efectivo de ellas. Si bien estas consideraciones forman parte del enfoque de las capacidades, particularmente desde las reflexiones de Sen sobre el funcionamiento, la implementación de este enfoque en nuestras legislaciones y políticas públicas no parece haber hecho eco de esto. Esta situación configura un problema relevante para este grupo de personas, si hemos aceptado a estas alturas la tesis de que este tipo de capacidades "sociales" constituyen, para el caso de la discapacidad mental, un contenido esencial de su ciudadanía porque guarda una relación directa con la etiología y tratamiento de su condición de salud.

Tal como he señalado, la discapacidad mental requiere considerar la asistencia como un asunto de ciudadanía activa, ya que son este tipo de variables las que han mostrado tener un peso significativo en su evolución y pronóstico, más allá de los aspectos biomédicos y farmacológicos del tratamiento tradicional de 
la deficiencia psíquica (Pérez, 2012; Sarraceno, 2003). Si consideramos que la asistencia de la salud mental y los derechos de igual ciudadanía corresponden a lo que entendemos por "justo", en el caso de la discapacidad mental se presentan también como contenidos de lo que tenemos por "bueno", ya que poseen un poder curativo tan importante que pueden disminuir el daño o deficiencia que ha dado origen a la condición de discapacidad de una persona. Considero que el análisis realizado puede constituir un punto firme para establecer que las consideraciones de justicia de quienes padecen discapacidades psíquicas requieren que sus capacidades de ciudadanía activa sean consideradas en una lista tentativa de las capacidades humanas básicas. En este sentido, me inclino más por aceptar provisionalmente la lista de capacidades básicas propuesta por Urquijo (2007), quien visualiza la capacidad de agencia como una de sus cinco capacidades humanas básicas, junto a las capacidades corporales, mentales, sociales y de singularidad. La capacidad de agencia es “...la que se establece por los funcionamientos de un ciudadano al actuar y provocar cambios e impactar en el mundo, y cuyos logros pueden juzgarse en función de sus propios valores y objetivos" (Urquijo, 2007 p. 34).

\section{Legislación y políticas públicas en Chile sobre PDM $^{3}$}

El Estado chileno adhiere a la gran mayoría de los pactos y estándares internacionales sobre legislación en salud mental. La adscripción a estos compromisos no es una mera formalidad pues somete a los estados a supeditar sus propias legislaciones locales a los principios y derechos consagrados por el derecho internacional. Especialmente desde la década de 1990, con el retorno del país a la democracia, es posible distinguir una intención sistemática de mejorar las condiciones de vida de las personas que padecen problemas de salud mental. Esta vocación de cambio se ha expresado en la generación de leyes y de políticas públicas relativas a la salud mental, en el marco de la adscripción del Estado y el Ministerio de Salud de Chile a las orientaciones internacionales existentes para la no discriminación, el respeto de los derechos humanos y la participación social de quienes padecen discapacidades psíquicas, como la Convención Sobre los Derechos de las Personas con Discapacidad, suscrita por nuestro país el año 2006 en Naciones Unidas.

Personas con discapacidad mental
En cuanto a los efectos de estas legislaciones en nuestros programas y políticas públicas de salud mental, es relevante considerar la creación, a la fecha, de dos Planes Nacionales de Salud Mental y Psiquiatría, el primero en 1993 y la segunda versión (vigente) en el año 2000. Dichos planes son el resultado de un importante esfuerzo por mejorar las condiciones de la atención de salud mental en el país y expresan, en el área de la salud mental, los fundamentos y orientaciones internacionales sobre los derechos de las personas con discapacidad. Dicho Plan está orientado en la convicción de que la salud mental no depende sólo "de factores biológicos inherentes a las personas y los grupos, sino también de las condiciones en que ellos viven" (Ministerio de Salud de Chile, 2001, p. 11), por lo que las acciones tendientes a favorecer la salud mental deben realizarse bajo un concepto de rehabilitación psicosocial y no sólo biomédico. El enfoque psicosocial o comunitario de la salud mental incorpora la idea de que "...es posible mejorar la calidad de vida de las personas con enfermedades mentales, ya que su deterioro no depende sólo de la enfermedad, sino también de las condiciones y comportamiento social de la familia y de la comunidad en que vive" (MINSAL, 2001, p. 12). En este contexto, el enfoque de la salud mental comunitaria impregna hoy el diseño de las políticas y programas públicos bajo la convicción de que los problemas de salud mental tienen su origen, más que en el acceso a los servicios de salud, en "factores psicosociales asociados al desempleo, las bajas remuneraciones, deterioro de las redes sociales de apoyo a la familia, escasa participación social, hacinamiento, menores niveles de educación, vecindarios más inseguros" (MINSAL, 2001, p. 21).

La situación de justicia de los denominados "discapacitados mentales" constituye un problema político, no sólo por la precariedad y marginación social en que todavía viven quienes padecen este tipo de enfermedades o trastornos mentales, sino porque nuestras leyes aún no protegen los más mínimos derechos humanos y civiles de esta parte de la ciudadanía. El único cuerpo legislativo específico vigente en Chile (Establece normas sobre deficientes mentales, 2008) está pronto a cumplir los treinta años, y obsoleto desde un punto de vista teórico, aunque se han realizado legislaciones de tipo complementario. En estas actualizaciones ha ocurrido la incorporación del paradigma de la discapacidad en las legislaciones relativas a la salud mental, instalándose progresivamente como una conceptualización hegemónica, al menos en términos legislativos y políticos. La revisión de estas legislaciones, incluso de sus complementos más actuales, evidencia que persisten 
importantes falencias en términos de la protección de derechos básicos en este grupo de personas, tales como la libertad, autonomía e igualdad de oportunidades, así como el acceso a la salud y la participación social (Ministerio de Salud de Chile, 2014; Nilo, 2011).

Por otra parte, en Chile las actualizaciones legislativas sólo se han desarrollado de manera complementaria, basadas en el paradigma de la discapacidad y en el principio de la integración social (Establece normas para la plena integración social de personas con discapacidad, 1994; Establece normas sobre igualdad de oportunidades e inclusión social de personas con discapacidad, 2015). La falta de una legislación específica y actualizada sobre salud mental es una falencia compartida con la mayoría de nuestros vecinos de la región, salvo Brasil y Argentina (OMS, 2013), por lo que el diseño y ejecución de una nueva legislación de salud mental para Chile debería considerar los desarrollos de estos países, particularmente de Argentina por ser la legislación más actualizada de Sudamérica y, por tanto, supuestamente más acorde con las actuales orientaciones internacionales sobre la materia. Una revisión comparada de estas legislaciones permitiría, no sólo evaluar la presencia del enfoque de la discapacidad como paradigma de la salud mental, sino también describir otros elementos relevantes que puedan aportar estas experiencias. Parece a todas luces necesario revisar estas discusiones, para pensar en una nueva y necesaria legislación sobre salud mental en Chile, una legislación efectivamente inclusiva y coherente con un paradigma social de la salud. Más allá de la discapacidad. En consideración de lo anterior es preciso recalcar que nos encontramos en el $75 \%$ de países con legislaciones de salud mental específicas y vigentes, aunque también entre el $49 \%$ de países con legislaciones promulgadas antes de 1990. Como se mencionó anteriormente, diversos estándares internacionales describen estas estadísticas subrayando el riesgo que implica para las personas el poseer legislaciones desactualizadas, ya que muchas veces exponen a los ciudadanos a situaciones de vulneración de sus derechos y no aseguran la protección de los mismos, como debiera ser su objetivo.

\section{Discusión: el desafío de una nueva legislación de salud mental para Chile}

La creación de una legislación de salud mental para Chile es una aventura imperiosa y arriesgada, ya que sus consecuencias afectarán directamente el rumbo de nuestra sociedad frente al problema de la discapacidad mental, y a que la posibilidad de enmendar errores u omisiones sólo puede hacerse efectiva con la creación de una nueva legislación en la materia, que reemplace a la ya existente. Sin embargo, es una tarea necesaria ya que el examen de la situación de justicia de este grupo de personas evidencia la persistencia de su exclusión de los derechos de ciudadanía, y de su marginación de la participación activa en la sociedad. El informe del recientemente creado Observatorio de Derechos Humanos de las personas con discapacidad mental en Chile (2014), permite confirmar este diagnóstico al señalar que las personas con discapacidad mental en nuestro país (2,18\% de la población) pertenecen en su mayoría a un nivel socioeconómico bajo. Por su parte, el informe del Ministerio de Salud de Chile sobre la materia (2014) sostiene que en la legislación vigente sobre salud mental persisten importantes falencias, como la negación de la capacidad jurídica, la negación de la capacidad de autonomía y las restricciones a la libertad de las personas con trastornos o discapacidades de tipo mental. Cabe destacar, que las falencias descritas en nuestra legislación se ubican precisamente en el terreno de lo que hemos venido entendiendo como ciudadanía activa, por lo que la corrección y modificación de la legislación vigente resulta de una urgencia evidente.

Sin embargo, la pretensión de este manuscrito ha sido establecer que para quienes presentan discapacidades mentales no basta una legislación que corrija en el texto las omisiones existentes, ni que incorpore en su listado de derechos las capacidades relativas a la agencia y la ciudadanía activa. La relevancia de estos aspectos o capacidades "sociales" en la etiología y tratamiento de la discapacidad mental requiere incorporar en la legislación mecanismos que fomenten y garanticen que dichas capacidades o derechos sean efectivamente puestos en funcionamiento por los individuos y la comunidad. Requiere por tanto asegurar que la condición de ciudadanía sea vivida de una manera activa por estas personas, más allá del respeto de sus derechos humanos básicos, incluyendo particularmente las consideraciones relativas a los principios de la autonomía y la igualdad de oportunidades. Si bien estos principios forman parte de los derechos de ciudadanía reconocidos en nuestras leyes como universales, para el caso de las personas con discapacidad mental se encuentran vetados ya en la legislación, poniendo en evidencia un paradigma sobre la justicia y la ciudadanía que los excluye ya que no puede dar cuenta de su diferencia. El enfoque de la discapacidad no parece capaz de hacerse cargo en términos políticos, epistemológicos 
o de diseño legislativo, de la situación de justicia de las personas con trastornos o enfermedades mentales. Se requiere por tanto de un paradigma o conceptualización de la salud mental que efectivamente incorpore los aspectos sociales en su real magnitud, y una legislación que garantice el ejercicio activo de estos derechos, a través de políticas públicas que promuevan la participación social y la asociatividad.

Las políticas y programas desarrollados por el Estado chileno en los últimos veinte años han significado mejoras importantes en la calidad de la asistencia de salud mental, pero respecto a los cambios sociales y las adecuaciones necesarias para asegurar su participación en la vida social ni las legislaciones ni las políticas públicas existentes parecen hacerse cargo. La situación de las organizaciones civiles de personas con discapacidad mental y sus familiares no son actualmente una responsabilidad asumida por el Estado, sino iniciativas particulares y aisladas, con escaso nivel de organización y participación y, por tanto, de influencia en la decisiones políticas relativas a la discapacidad mental. Podemos afirmar, en consecuencia, que sus capacidades de agencia (Urquijo, 2007) se encuentran severamente vulneradas, muy por debajo del umbral mínimo intuitivo, ya que las posibilidades de los discapacitados mentales de provocar cambios e impactar en su mundo deben incorporar estrategias colectivas de organización para influir realmente en su entorno y participar activamente en la resolución de sus problemas. Su nivel de marginación social es severo, ya que su discapacidad los ha excluido sistemáticamente del sistema educativo y laboral, así como de la posibilidad de establecer relaciones sociales y afectivas significativas, en la mayoría de los casos.

El ejercicio de sus capacidades de ciudadanía activa requiere de políticas públicas que aseguren aspectos como la inserción laboral y educativa, ya que en el caso de quienes padecen discapacidades psíquicas se encuentran vulneradas todas o la mayoría de sus capacidades básicas, pero requiere, sobretodo, de iniciativas que fomenten sus capacidades autónomas de participación como ciudadanos y no sólo como asistidos o pacientes (MINSAL, 2014). El foco aquí debería estar puesto en evitar las políticas asistencialistas y fomentar la autonomía y las capacidades de organización ciudadana de estas personas y sus familiares, ya que la ciudadanía activa no puede pensarse seriamente como una tarea del estado, sino que refiere más bien al protagonismo de la comunidad y los ciudadanos (Cortina, 1997). Sin embargo, el empoderamiento respecto de sus capacidades de ciudadanía activa requiere el desarrollo de procesos que no pueden llevar adelante por su propia cuenta, precisamente a causa del alto nivel de dependencia y marginalidad en que han existido. No es posible soslayar la responsabilidad que le cabe al Estado en este asunto, así como a toda la sociedad.

Finalmente, la nueva legislación chilena sobre la discapacidad requiere la implementación de un importante debate en nuestra sociedad, pero sobre todo precisa la incorporación de la perspectiva de los principales actores involucrados: los discapacitados mentales. La incorporación de las personas con discapacidad mental y sus familiares en los cambios y avances legislativos es fundamental, no sólo por la valiosa experiencia y conocimiento que pueden aportar sobre este problema, sino porque su participación es necesaria como garantía de su condición de igual ciudadanía. Si hemos aceptado la premisa de que quienes padecen discapacidades mentales (y sus familiares) pueden y necesitan ejercer su ciudadanía de manera activa, entonces debemos hacernos cargo honestamente de que nadie mejor que ellos puede representar adecuadamente sus demandas e intereses.

Además, si constatamos que son precisamente las capacidades de agencia y ciudadanía activa las que permanecen ausentes en la legislación vigente, la participación de este grupo de personas y sus familiares, así como de las diversas organizaciones de la sociedad civil que se preocupan del problema de la discapacidad debería ser una prioridad, ya que son ellos los interlocutores mejor calificados para hablar del ejercicio efectivo de su ciudadanía. La inclusión de los discapacitados y la sociedad civil en la legislación sobre la discapacidad ha sido asumida por primera como una prioridad en la historia de Naciones Unidas en el proceso de creación de la Convención Sobre los Derechos de las Personas con Discapacidad el año 2006, señalando por esto un verdadero hito en el tratamiento de los problemas de justicia de quienes padecen discapacidades. La reciente promulgación de esta Convención en nuestro país debiera comprometernos entonces en seguir este ejemplo, asumiendo la oportunidad de mejorar las condiciones de justicia de los discapacitados mentales y sus familiares de una manera realmente inclusiva.

Nuestro desafío actual de contar con una legislación de salud mental acorde a los estándares internacionales es una tarea urgente y compleja, que requiere nutrirse de diversas fuentes y experiencias para constituir un piso firme sobre el que trabajar los próximos años. Pero diseñar una nueva ley de salud mental que incorpore efectivamente la perspectiva de los propios discapacitados mentales y sus familiares es un desafío aún 
mayor, ya que implica cambiar la forma en que nuestra sociedad visualiza las posibilidades de ciudadanía de estas personas. Ello debido a que, como ya hemos establecido en la discusión, si bien representantes que ellos no han escogido pueden ayudar a resolver la situación de sus derechos y justicia, difícilmente podrán representarlos, ya que con certeza no padecen una discapacidad de tipo mental.

\section{Referencias}

Cortina, A. (1986). Ética Mínima. Introducción a la filosofía práctica. Madrid: Tecnos.

Cortina, A. (1997). Ciudadanos del Mundo. Hacia una Teoría de la Ciudadanía. Madrid: Alianza.

Foucault, M. (1967). Historia de la Locura en la Época Clásica. México, D.F.: Fondo de Cultura Económica.

Ley 18.600. "Establece Normas Sobre Deficientes Mentales". Biblioteca del Congreso Nacional. Recuperado de http://www. leychile.cl/Navegar?idNorma $=29991$

Ley 19.284. "Establece Normas para la Plena Integración Social de las Personas con Discapacidad". Biblioteca del Congreso Nacional. Recuperado de http://www.leychile.cl/Navegar/scripts/ob tienearchivo?id=recursoslegales/10221.3/34115/1/HL19284.pdf

Ley 20.422. "Establece Normas sobre Igualdad de Oportunidades e Inclusión Social de Personas con Discapacidad”. Diario oficial de la República de Chile, Santiago, Chile, Febrero de 2010. Recuperado de http://www.senadis.gob.cl/pag/177/620/ley_n20422

Loubat, M., Gárate, R. y Cuturrufo, N. (2014). Investigaciones en rehabilitación cognitiva para pacientes con esquizofrenia (20042013): una revisión temática en beneficio de propuestas para etapas iniciales del trastorno. Revista Chilena de Neuro-Psiquiatría, 52(3), 213-227. Recuperado de http://www.scielo.cl/pdf/rchnp/ v52n3/art07.pdf

Ministerio de Salud de Chile. (2000). Plan Nacional de Salud Mental y Psiquiatría. Recuperado de http://www.psiquiatriasur.cl/portal/ modules/wfdownloads/visit.php?cid $=27 \&$ lid $=302$

Ministerio de Salud de Chile (2014). Evaluación del Sistema de Salud Mental en Chile (Segundo Informe). Recuperado de http://www. observatoriodiscapacidadmental.cl/wp-content/uploads/2014/08/ WHO-AIMS-II-SISTEMA-SALUD-MENTAL-DE-CHILE-2014. pdf

Nilo, R. (2011). Ciudadanía Activa y Discapacidad Psíquica. Un análisis crítico de la teoría de John Rawls frente a la situación de justicia de los pacientes psiquiátricos. Sarrebruck: Académica Española.
Nussbaum, M. (2007). Las Fronteras de la Justicia. Consideraciones Sobre la Exclusión. Barcelona: Paidós.

Observatorio de Derechos Humanos de las Personas con Discapacidad Mental. (2014). Derechos Humanos de las Personas con Discapacidad Mental: Diagnóstico de la Situación en Chile. Recuperado de http://www.observatoriosaludmental.cl/wp-content/ uploads/2014/05/informe-ODDHHPDM-final.pdf

Organización de las Naciones Unidas. (1993). Normas Uniformes para la Equiparación de Oportunidades para las Personas con Discapacidad. Recuperado de http://www.un.org/spanish/disabilities/default.asp?id=498

Organización de las Naciones Unidas. (2006). Convención Sobre los Derechos de las Personas con Discapacidad. Recuperado de http://www.un.org/esa/socdev/enable/documents/tccconvs.pdf

Organización Mundial de la Salud. (2001). Clasificación Internacional del Funcionamiento, de la Discapacidad y de la Salud. Recuperado de http://conadis.gob.mx/doc/CIF_OMS.pdf

Organización Mundial de la Salud. (2006). Manual de Recursos de la OMS sobre Salud Mental, Derechos Humanos y Legislación. Ginebra: Ediciones de la OMS.

Organización Mundial de la Salud. (2013). WHO-AIMS: Informe Sobre los Sistemas de Salud Mental en América Latina y El Caribe. Recuperado de http://www.paho.org/per/images/stories/ FtPage/2013/WHO-AIMS.pdf

Ovejero, F., Martí, J. \& Gargarella, R. (Eds.) (2004). Nuevas Ideas Republicanas. Autogobierno y Libertad. Barcelona: Paidós.

Pérez, C. (2012). Una Nueva Antipsiquiatría. Crítica y conocimiento de las técnicas de control psiquiátrico. Santiago: LOM.

Rawls, J. (1971). Teoría de la Justicia. México, D.F.: Fondo de Cultura Económica.

Rawls, J. (2001). La Justicia Como Equidad: Una Reformulación. España: Editorial Paidós.

Sarraceno, B. (2003). La Liberación de los Pacientes Psiquiátricos: De la rehabilitación psicosocial a la ciudadanía posible. México, D.F.: Pax.

Sen, A. (1995). Nuevo Examen de la Desigualdad. Madrid: Alianza. Toboso, M. \& Arnau, M. (2008). La discapacidad dentro del enfoque de capacidades y funcionamientos de Amartya Sen. Araucaria, Revista Iberoamericana de Filosofía, Política y Humanidades, 10(20), 64-94. Recuperado de http://hdl.handle.net/10261/23277

Urquijo, M. (2007). La Ciudadanía Reexaminada. Un análisis a la ciudadanía democrática desde el enfoque de las capacidades humanas. Cali: Universidad del Valle. 